\title{
Calculation of Higgs Self-Coupling Constant for One-Loop and Two-Loop Effective Potentials and Their Perturbative Validity
}

\author{
Partha Pratim Pal \\ Bolpur Sikshaniketan Ashram Vidyalaya, West Bengal, Bolpur, Birbhum, India
}

Email address:

partha4321@yahoo.com

To cite this article:

Partha Pratim Pal. Calculation of Higgs Self-Coupling Constant for One-Loop and Two-Loop Effective Potentials and Their Perturbative Validity. American Journal of Physics and Applications. Vol. 3, No. 2, 2015, pp. 63-67. doi: 10.11648/j.ajpa.20150302.18

\begin{abstract}
Although the Standard Model has been very successful in explaining the present data, it is unable to express the value of Higgs self coupling constant. It is a free parameter of the theory. Recently a Higgs like particle is reported to be discovered at CMS and ATLAS experiments at CERN LHC with a mass of about $125 \mathrm{GeV}$. The purpose of this paper is to calculate the value of Higgs self coupling constant in the Standard Model for one and two-loops Higgs potential. Considering the value of Higgs mass as $125 \mathrm{GeV}$ we obtain the value of Higgs self coupling constant as 0.1373 for both one and two-loops Higgs potential. We also find that the condition for perturbative validity given by Miller is satisfied, when the Higgs mass is calculated perturbatively from the effective potential given by the massless $\varphi^{4}$ theory at one and two-loop level.
\end{abstract}

Keywords: Standard Model, Higgs Self-Coupling Constant, LHC, Perturbative Validity

\section{Introduction}

The Standard Model (SM) of particle physics represents an example of calculable quantum field theory. This was achieved after years of efforts of several scientists like S. L. Glashow, S. Weinberg, A. Salam for understanding the theory of electro-weak interaction [1].The Standard Model, is being used to describe the fundamental particles (quarks and leptons) and forces. It works extremely well. It has successfully predicted and accounted for the observations of experiments at LEP, the Tevatron at Fermilab and other particle physics experiments. However, it requires the existence of a particle, known as the Higgs boson [2,3].The search for Higgs boson is one of the main goals of present and future high energy colliders [4].The observation of this particle is very much important for the understanding of the fundamental interactions among the quarks and leptons, as well as the generation of masses of elementary particles. Actually, in order to understand the unification of wellestablished electro-magnetic and weak interaction phenomena, the existence of at least one iso-doublet scalar field is needed for the generation of fermion and weak gauge boson masses. In SM, where one iso-doublet scalar field is used, three Goldstone bosons among the four degrees of freedom absorb the longitudinal component of the massive
$W^{ \pm}, \mathrm{Z}$ gauge bosons and the degree of freedom that is left over corresponds to a physical scalar particle, called the Higgs boson [2].

The Higgs boson has been searched at LEP, and its mass should be greater than $114 \mathrm{GeV}$ [3]. . Recently a Higgs like particle is reported to be discovered at CMS and ATLAS experiments at CERN LHC [5] with a mass of about 125 $\mathrm{GeV}$. In the SM the properties of the Higgs particle is uniquely determined, once its mass is fixed. Unfortunately the Higgs self-coupling constant is a free parameter of the theory.

The potential for scalar field is given by $\mathrm{V}(\varphi)=-\mu^{2} \varphi^{+}$ $\varphi+\lambda\left(\varphi^{+} \varphi\right)^{2}$. For positive values of $\mu^{2}$ and $\lambda$, we have spontaneous symmetry breakdown as the scalar develops a vacuum expectation value and $\left\langle\varphi_{>_{0}}=<0|\quad \varphi| \quad 0>\neq 0\right.$ with $\mathrm{v}=\left(\mu^{2} / \lambda\right)^{1 / 2}$.The vacuum expectation value has the size $\mathrm{v}=$ $2^{-1 / 4} \mathrm{G}_{\mathrm{F}}^{-1 / 2} \approx 246 \mathrm{GeV}$.

Although there is some knowledge of $\mathrm{v}$, at present there is no information on the quartic coupling $\lambda$. If $\lambda$ is too small, the symmetry breaking vacuum will be unstable producing a lower bound e.g. the Linde-Weinberg bound. Here the oneloop contributions (specially those due to gauge-boson loop) to the effective potential of path integral formalism of 
quantum field theory becomes relatively important .The details of the generalization of these calculations for $\mathrm{SU}(2)$ $\times \mathrm{U}(1)$ is given in the celebrated work of Coleman and Weinberg [6], Weinberg [7], Linde and Weinberg [8].

It is important that the effective potentials used in the one or two loop descriptions should be free from false perturbative minima. This is because it has been suggested by Miller [9] that an effective potential evaluated perturbatively can possess false minima, (i.e. non existent minima) because of the approximation method itself. So in this section, we have tried to test the effective potentials used for calculation of Higgs mass for perturbative validity following the work of Miller [7].The effective potential choosen here considers both one and two- loop Higgs pontential and includes the effect of scalar, vector and fermion loops to the potential [10].

Miller wrote the two-loop effective potential by redefining the subtraction point $(\mu)$ in a variant the minimal subtraction to obtain [9]

$$
\mathrm{V}\left(\varphi_{\mathrm{cl}}\right)=\frac{\lambda \varphi_{c l}{ }^{4} S\left(\varphi_{c l}\right)}{4 !}
$$

where $\mathrm{S}\left(\varphi_{\mathrm{cl}}\right)$ is a perturbative series, $\varphi_{\mathrm{cl}}$ is the classical field $\left(\varphi_{\mathrm{cl}}=\frac{\delta W[J]}{\delta J}\right.$,

$\mathrm{W}=$ generator of connected Green function). Using the short hand notations $\alpha=\frac{\lambda}{16 \pi^{2}}$ and $1 \varphi=\ln \left(\frac{\varphi_{c l}{ }^{2}}{\mu^{2}}\right)$ Miller showed that one could resumeS( $\left.\varphi_{\mathrm{cl}}\right)$ perturbation series at $\mathrm{u}$ loop order as

$$
\mathrm{S}\left(\varphi_{\mathrm{cl}}\right)=\sum_{m=0}^{u}(\alpha 1 \varphi)^{\mathrm{m}} \mathrm{C}_{\mathrm{m}}
$$

Now, spontaneous symmetry breaking is indicated by a non-zero (minimal) solution to

$$
\left.\frac{d V\left(\varphi_{c l}\right)}{d \varphi_{c l}}\right|_{\varphi_{c l}=\sigma}=0
$$

On minimizing the effective potential if we find a non-zero vacuum expectation value, then the theory under consideration is said to be spontaneously broken. There exists a problem with the perturbative approach. The question is not about the reliability of the result but whether the indicated spontaneous breaking actually exists or not. In fact, the condition for actual existence of spontaneous symmetry breaking is $|\alpha 1 \sigma|<1$. Actually the approximate nature of perturbation theory can generate false minima to effective potentials. So we try to test the effective potential which is used for calculating Higgs mass for spontaneous symmetry breaking.

The purpose of this paper is to extend the studies on Higgs self-coupling constantby incorporating the following modifications: (i) our calculation includes the effects of the scalar and fermion loops in addition to the vector loop;(ii) the study is extended to two- loop correction to the potential as given in [9]; (iii) The perturbative validity for one and two- loops Higgs potential, is tested by proving that $\left|\alpha l_{\sigma}\right|<1$ for $\mu^{2} \neq 0$.

The paper is organized as follows: in section 2 the theory for calculating Higgs self-coupling constant for one-loop and two-loop effective potentials is given. The theory of perturbative validity for one and two-loop Higgs potentials are given in section 3 . The results and discussions are given in section 4 .

\section{Theory for Calculating Higgs Self-Coupling Constant for One-Loop and Two-Loop Effective Potentials}

The full effective potential at one-loop level which include the effect of scalar, vector and fermion loops is given by

$$
V(\varphi)=V_{0}(\varphi)+V_{1}(\varphi)=-\mu^{2} \varphi^{2}+\lambda \varphi^{4}+C \varphi^{4} \ln \left(\frac{\varphi^{2}}{M^{2}}\right)
$$

Where

$$
C=\frac{1}{16 \pi^{2} v^{4}}\left(3 \sum m_{v}^{4}+m_{s}^{4}-4 \sum m_{f}^{4}\right)
$$

Here, $\mathrm{M}$ is an arbitrary mass parameter, $\mu^{2}$ is defined as the mass squared term in $\lambda \varphi^{4}$ theory.

Hence, the condition $\frac{\partial V}{\partial \phi} \mid=0$ gives

$$
\begin{gathered}
\varphi=\frac{v}{\sqrt{2}} \\
-\mu^{2}+\lambda v^{2}+C v^{2}\left\{\ln \left(\frac{v^{2}}{2 M^{2}}\right)+\frac{1}{2}\right\}=0
\end{gathered}
$$

Thus the Higgs mass is given by

$$
\begin{gathered}
\mathrm{m}^{2}=\frac{1}{2} \frac{\partial^{2} V}{\partial \varphi^{2}} \mid=2 \lambda \mathrm{v}^{2}+\mathrm{Cv}^{2}\left\{2 \ln \left(\frac{v^{2}}{2 M^{2}}\right)+3\right\} \\
\varphi=\frac{v}{\sqrt{2}}
\end{gathered}
$$

Where $\mu^{2}$ is substituted from eq.(3).

Hence, from equation (4) we can write the expression for $\lambda$ as

$$
\lambda=\frac{1}{2 v^{2}}\left[m_{H}^{2}-C v^{2}\left\{2 \ln \left(\frac{v^{2}}{2 M^{2}}\right)+3\right\}\right]
$$

The Higgs potential containing the two-loop correction term [7] to the full potential is

$$
V=-\mu^{2} \varphi^{2}+\lambda \varphi^{4}+C \varphi^{4} \ln \left(\frac{\varphi^{2}}{M^{2}}\right)+\frac{3 \lambda^{3} \varphi^{4}}{56\left(16 \pi^{2}\right)^{2}}\left(84 l_{\varphi}^{2}-176 l_{\varphi}-137\right)
$$

Where 


$$
l_{\varphi}=\ln \left(\frac{\varphi^{2}}{M^{2}}\right)
$$

Using eq.(5), we calculate $\frac{\partial V}{\partial \phi}$ and the condition $\frac{\partial V}{\partial \phi} \mid=0$

$$
\mu^{2}=\lambda v^{2}+C v^{2}\left\{\ln \left(\frac{v^{2}}{2 M^{2}}\right)+\frac{1}{2}\right\}+\frac{3 \lambda^{3} v^{2}}{56\left(16 \pi^{2}\right)^{2}}\left[84\left\{\ln \left(\frac{v^{2}}{2 M^{2}}\right)\right\}^{2}-176 \ln \left(\frac{v^{2}}{2 M^{2}}\right)-137\right]+\frac{3 \lambda^{3} v^{2}}{224\left(16 \pi^{2}\right)^{2}}\left[336 \ln \left(\frac{v^{2}}{2 M^{2}}\right)-352\right]
$$

Hence, with the inclusion of two-loop correction term the Higgs mass is given by

$$
\begin{gathered}
m_{H}{ }^{2}=-\mu^{2}+3 \lambda v^{2}+3 C v^{2} \ln \left(\frac{v^{2}}{2 M^{2}}\right)+\frac{7}{2} \mathrm{Cv}^{2}+\frac{9 \lambda^{3} v^{2}}{56\left(16 \pi^{2}\right)^{2}}\left[84\left\{\ln \left(\frac{v^{2}}{2 M^{2}}\right)\right\}^{2}-176 \ln \left(\frac{v^{2}}{2 M^{2}}\right)-137\right]+\frac{21 \lambda^{3} v^{2}}{224\left(16 \pi^{2}\right)^{2}}\left[336 \ln \left(\frac{v^{2}}{2 M^{2}}\right)-\right. \\
352]+\frac{9 \lambda^{3} v^{2}}{\left(16 \pi^{2}\right)^{2}}
\end{gathered}
$$

Substituting the value of $\mu^{2}$ from eq. (8) in eq. (9), the expression for Higgs mass comes out to be

$$
\begin{gathered}
m_{H}{ }^{2}=2 \lambda v^{2}+C v^{2}\left\{2 \ln \left(\frac{v^{2}}{2 M^{2}}\right)+3\right\}+\frac{3 \lambda^{3} v^{2}}{28\left(16 \pi^{2}\right)^{2}}\left[84\left\{\ln \left(\frac{v^{2}}{2 M^{2}}\right)\right\}^{2}-176 \ln \left(\frac{v^{2}}{2 M^{2}}\right)-137\right]+\frac{9 \lambda^{3} v^{2}}{112\left(16 \pi^{2}\right)^{2}}\left[336 \ln \left(\frac{v^{2}}{2 M^{2}}\right)-352\right]+ \\
\frac{9 \lambda^{3} v^{2}}{112\left(16 \pi^{2}\right)^{2}}
\end{gathered}
$$

This equation will be used to calculate the value of $\lambda$ in two-loop effective potential.

\section{Theory of Perturbative Validity for One and Two Loops Higgs Potentials}

The purpose of this section is to prove the perturbative validity of both one and two-loop Higgs potentials, which includes the effects of scalar,vector and fermion loops. Here we obtain the expressions for $\left|\alpha l_{\sigma}\right|$ for one and two- loop Higgs potential separately which will be used to calculate the numerical value of $\left|\alpha l_{\sigma}\right|$ for the justification of perturbativevalidity. This gives an illustration of a general method which would establish whether the indicated spontaneous breaking actually exists or not.

\section{1. $\alpha$ l $\sigma$ for 1-loop}

We start with

$$
\mathrm{V}\left(\varphi_{c l}\right)=\lambda \varphi_{c l}^{4}+\mathrm{C} \varphi_{c l}^{4} \ln \left(\frac{\varphi_{c l}^{2}}{M^{2}}\right)-\mu^{2} \varphi_{c l}^{2}
$$

Where $C=\frac{1}{16 \pi^{2} v^{4}}\left(\sum 3 m_{v}^{4}+m_{s}^{4}-4 \sum m_{f}^{4}\right)$

Here the effect of vector,scalar and fermion loops are taken into account.

We can write equation (1) as

$$
\mathrm{V}\left(\varphi_{c l}\right)=\lambda \varphi_{c l}^{4} \mathrm{~S}\left(\varphi_{\mathrm{cl}}\right)
$$

where

$$
\mathrm{S}\left(\varphi_{c l}\right)=1-\frac{\mu^{2}}{\lambda \phi_{c l}^{2}}+\frac{C}{\lambda} \ln \left(\frac{\varphi_{c l}^{2}}{M^{2}}\right)
$$

Or,

$$
\mathrm{S}(\sigma)=1-\frac{\mu^{2}}{\lambda \sigma^{2}}+\frac{C}{\lambda} 1_{\sigma}
$$

Here $l_{\sigma}=\ln \left(\frac{\sigma^{2}}{M^{2}}\right)$, so that

$$
\frac{d S(\sigma)}{d l_{\sigma}}=\frac{C}{\lambda}+\frac{\mu^{2}}{\lambda \sigma^{2}}
$$

Since,

$$
2 \mathrm{~S}(\sigma)+\frac{d S(\sigma)}{d l_{\sigma}}=0
$$

we get, by substituting the values of $\mathrm{S}(\sigma)$ and $\frac{d S(\sigma)}{d l_{\sigma}}$ in equation (15), the following relation

$$
\alpha l_{\sigma}=\frac{\lambda \mu^{2}}{32 \pi^{2} C \sigma^{2}}-\frac{\lambda}{32 \pi^{2}}-\frac{\lambda^{2}}{16 \pi^{2} C}
$$

\subsection{Calculation of $\alpha$ l $\sigma$ for 2-loop}

The 2-loop potential is

$$
\mathrm{V}\left(\varphi_{\mathrm{cl}}\right)=-\mu^{2} \varphi_{c l}^{2}+\lambda \varphi_{c l}^{4}+C \varphi_{c l}^{4} \ln \left(\frac{\varphi_{c l}^{2}}{M^{2}}\right)+\frac{3 \lambda^{3} \varphi_{c l}^{4}}{56\left(16 \pi^{2}\right)^{2}}\left[84 l_{\varphi}^{2}-176 l_{\varphi}-137\right]
$$


Where

$$
C=\frac{1}{16 \pi^{2} \mathrm{v}^{4}}\left(\sum 3 m_{v}^{4}+m_{s}^{4}-4 \sum m_{f}^{4}\right)
$$

and

$$
\mathrm{S}\left(\varphi_{\mathrm{cl}}\right)=\frac{\mathrm{V}\left(\varphi_{\mathrm{cl}}\right)}{\lambda \varphi_{\mathrm{cl}}^{4}}=1-\frac{\mu^{2}}{\lambda \varphi_{\mathrm{cl}}^{2}}+\frac{\mathrm{Cl} \varphi_{\varphi}}{\lambda}+\frac{3 \lambda^{2}}{56\left(16 \pi^{2}\right)^{2}}\left[84 \mathrm{l}_{\varphi}^{2}-176 \mathrm{l}_{\varphi}-137\right]
$$

Hence

$$
\mathrm{S}(\sigma)=1-\frac{\mu^{2}}{\lambda \sigma^{2}}+\frac{C l_{\sigma}}{\lambda}+\frac{3 \lambda^{2}}{56\left(16 \pi^{2}\right)^{2}}\left[84 l_{\sigma}^{2}-176 l_{\sigma}-137\right]
$$

so that

$$
\frac{d S(\sigma)}{d l_{\sigma}}=\frac{C}{\lambda}+\frac{\mu^{2}}{\lambda \sigma^{2}}+\frac{3 \lambda^{2}}{56\left(16 \pi^{2}\right)^{2}}\left[168 l_{\sigma^{-176}}\right]
$$

Now, equation (15) gives

$$
9\left(\alpha l_{\sigma}\right)^{2}+\left(\frac{32 \pi^{2} C}{\lambda^{2}}-\frac{69 \alpha}{7}\right) \alpha l_{\sigma}+2-\frac{\mu^{2}}{\lambda \sigma^{2}}+\frac{C}{\lambda}-\left(\frac{411}{28}+\frac{66}{7}\right) \alpha^{2}=0
$$

Writing $\mathrm{X}=\alpha l_{\sigma}$, equation (22) can be written as

$$
\mathrm{AX}^{2}+\mathrm{BX}+\mathrm{C}^{\prime}=0
$$

$$
\mathrm{X}=\frac{-B \pm \sqrt{B^{2}-4 A C^{\prime}}}{2 A}
$$

where $\mathrm{A}=9 ; \mathrm{B}=\frac{32 \pi^{2} C}{\lambda^{2}}-69 \frac{\alpha}{7}$ and $\mathrm{C}^{\prime}=2-\frac{\mu^{2}}{\lambda \sigma^{2}}+\frac{C}{\lambda}$ $\left(\frac{411}{28}+\frac{66}{7}\right) \alpha^{2}$

The solution to eq.(23) is

$$
\begin{gathered}
v=246 \mathrm{GeV}, m_{t}=175 \mathrm{GeV}, m_{b}=5 \mathrm{GeV}, M_{w}=80 \mathrm{GeV}, m_{z}=91 \mathrm{GeV}, M=180 \mathrm{GeV}, \sigma=246 \mathrm{GeV} \text { and } \mathrm{C}= \\
-0.00570118 \text { for } m_{s}=0
\end{gathered}
$$

Considering the value of Higgs mass to be $125 \mathrm{GeV}$, we get the value of $\lambda$ from equations (4) and (10) for one-loop and two-loop effective potentials respectively. In both oneloop and two-loop cases the value of $\lambda$ comes out to be 0.1373 . It is worth mentioning that the value of $\lambda$ is nearly independent of M. I also find that the effect of two-loop contribution is negligibly small. Furthermore, while calculating the value of $\left|\alpha l_{\sigma}\right|$ from equations (16) and (24) for one-loop and two-loop effective potentials respectively, it is observed that $\left|\alpha l_{\sigma}\right|<1$. So, the perturbative validity of the present result is established.

\section{References}

[1] S. L. Glashow, 1961 Nucl. phys. 22, 579; A. Salam, 1968, in Elementary Particle Theory, Almquist and Forlag, Stockholm; A. Salam and J. C. Ward, 1964 Phys. Lett, 13, 168; S. Weinberg, 1967 Phys. Rev. Lett. 19, 1264; Cheng T. P. and Li L.F., Gauge Theory Of Elementary Particle Physics, Clarendon Press, Oxford, 1984. P396.

[2] Higgs P.W. (1964) Phys. Rev. Lett. 12, 132; (1966) Phys. Rev.

$$
145,1156
$$

[3] Englert F and Brout R 1964 Phys.Rev.Lett.13, 321; Guralnik G S, Hagen C R and Kibble T W B 1964 Phys.Rev.Lett. 13, 585 .

[4] LEP Higgs Working Group, CERN - EP / 2001-055; Abbiendi, G, Phys.Lett.B565 (2003)61; Review of Higgs Physics at the LHC, V. Drollinger, hep-ph/0405026, 2004.

[5] Large Hadron Collider-Wikipedia, the free encyclopaedia en.wikipedia.org/wiki/Large-Hadron-Higgs-like Particle Discovered at CERN; Kane G,Pierce A(ed)2008 Perspective on LHC physics (Singapore:World Science)

[6] Coleman S. and Weinberg E. (1973) Phys. Rev. D7, 1888

[7] Weinberg S. (1979) Phys. Lett. 82B, 387

[8] Linde A. D. (1976) Sov. Phys. JETPLett. 23, 64; Weinberg S. (1976) Phys. Rev. Lett. 36, 294.

[9] Miller Robert D C (1983). J.Phys. G ,Nucl .Phys. 9 , 697

[10] Sher M, Phys. Rep. (1989) 179, 299. 